\title{
Review and scientific prospects of high-contrast optical stellar interferometry
}

Defrère, D., Absil, O., Berger, J.-P., Danchi, W. C., Dandumont, C., et al.

D. Defrère, O. Absil, J.-P. Berger, W. C. Danchi, C. Dandumont, F.

Eisenhauer, S. Ertel, T. Gardner, A. Glauser, P. Hinz, M. Ireland, J. Kammerer, S. Kraus, L. Labadie, S. Lacour, R. Laugier, J. Loicq, G. Martin, F. Martinache, M. A. Martinod, B. Mennesson, J. Monnier, B. Norris, M. Nowak, J. U. Pott, S. P. Quanz, E. Serabyn, J. Stone, P. Tuthill, J. Woillez, "Review and scientific prospects of high-contrast optical stellar interferometry," Proc. SPIE 11446, Optical and Infrared Interferometry and Imaging VII, 114461J (13 December 2020); doi: $10.1117 / 12.2561505$ 


\section{Review and scientific prospects of high-contrast optical stellar interferometry}

D. Defrère ${ }^{a}$, O. Absil ${ }^{b, \star}$, J.-P. Berger ${ }^{c}$, W.C. Danchi ${ }^{d}$, C. Dandumont ${ }^{b}$, F. Eisenhauer ${ }^{e}$, S. Ertel ${ }^{f}$, T. Gardner ${ }^{g}$, A. Glauser ${ }^{h}$, P. Hinz ${ }^{i}$, M. Ireland ${ }^{j}$, J. Kammerer $^{k}$, S. Kraus ${ }^{l}$, L. Labadie ${ }^{m}$, S. Lacour ${ }^{n}$, R. Laugier ${ }^{a}$, J. Loicq ${ }^{b}$, G. Martin ${ }^{c}$, F. Martinache ${ }^{o}$, M.A. Martinod ${ }^{p}$, B. Mennesson ${ }^{r}$, J. Monnier ${ }^{g}$, B. Norris ${ }^{p}$, M. Nowak ${ }^{s}$, J.U. Pott ${ }^{q}$, S.P. Quanz ${ }^{h}$, E. Serabyn ${ }^{r}$, J. Stone ${ }^{t, \star \star}$, P. Tuthill ${ }^{p}$, and J. Woillez ${ }^{k 1}$

${ }^{a}$ Institute of Astronomy, KU Leuven, Celestijnenlaan 200D, 3001, Leuven, Belgium

${ }^{b}$ Space sciences, Technologies \& Astrophysics Research (STAR) Institute, University of Liège, Liège, Belgium

${ }^{c}$ Univ. Grenoble Alpes, CNRS, IPAG, 38000 Grenoble, France

${ }^{d}$ NASA Goddard Space Flight Center, Exoplanets \& Stellar Astrophysics Laboratory, Greenbelt, USA

${ }^{e}$ Max Planck Institute for extraterrestrial Physics, Giessenbachstraße 1, 85748 Garching, Germany

${ }^{f}$ Steward Observatory, Department of Astronomy, University of Arizona, Tucson, Arizona, USA

${ }^{g}$ University of Michigan, Ann Arbor, United States

${ }^{h}$ Eidgenössische Technische Hochschule (ETH) Zurich, Institute for Particle Physics and Astrophysics, Zurich, Switzerland

${ }^{i}$ University of California, Santa Cruz, Santa Cruz, CA 95064, USA

${ }^{j}$ Research School of Astronomy and Astrophysics, Australian National University, Canberra, ACT 2611, Australia

${ }^{k}$ European Southern Observatory, Karl-Schwarzschild-Straße 2, 85748 Garching, Germany

${ }^{l}$ School of Physics and Astronomy, University of Exeter, Exeter, United Kingdom

${ }^{m}$ I. Physikalisches Institut, Universität zu Köln, Zülpicher Str. 77, 50937 Cologne, Germany

${ }^{n}$ LESIA, Observatoire de Paris, PSL Research University, 92195 Meudon Cedex, France

${ }^{\circ}$ Laboratoire Lagrange, Université Côte d'Azur, Observatoire de la Côte d'Azur, CNRS,

Boulevard de l'Observatoire, CS 34229, 06304, Nice, France

${ }^{p}$ University of Sydney, Sydney, Australia

${ }^{q}$ Max Planck Institute for Astronomy, Königstuhl 17, 69117 Heidelberg, Germany

${ }^{r}$ Jet Propulsion Laboratory, California Institute of Technology, Pasadena, CA 91109, USA

${ }^{s}$ Institute of Astronomy, Madingley Road, Cambridge CB3 0HA, UK

${ }^{t}$ Naval Research Laboratory, Remote Sensing Division, 4555 Overlook Ave SW, Washington, DC 20375, USA

\footnotetext{
ABSTRACT

High-contrast optical stellar interferometry generally refers to instruments able to detect circumstellar emission at least a few hundred times fainter than the host star at high-angular resolution (typically within a few $\lambda / \mathrm{D}$ ). While such contrast levels have been enabled by classical modal-filtered interferometric instruments such as

$\star$ FNRS Research Associate.

$\star \star$ Hubble Fellow

Optical and Infrared Interferometry and Imaging VII, edited by Peter G. Tuthill, Antoine Mérand,

Stephanie Sallum, Proc. of SPIE Vol. 11446, 114461J · (C) 2020 SPIE

CCC code: $0277-786 \mathrm{X} / 20 / \$ 21 \cdot$ doi: $10.1117 / 12.2561505$ 
VLTI/PIONIER, CHARA/FLUOR, and CHARA/MIRC the development of instruments able to filter out the stellar light has significantly pushed this limit, either by nulling interferometry for on-axis observations (e.g., PFN, LBTI, GLINT) or by off-axis classical interferometry with VLTI/GRAVITY. Achieving such high contrast levels at small angular separation was made possible thanks to significant developments in technology (e.g., adaptive optics, integrated optics), data acquisition (e.g., fringe tracking, phase chopping), and data reduction techniques (e.g., nulling self-calibration). In this paper, we review the current status of high-contrast optical stellar interferometry and present its key scientific results. We then present ongoing activities to improve current ground-based interferometric facilities for high-contrast imaging (e.g., Hi-5/VIKING/BIFROST of the ASGARD instrument suite, GRAVITY+) and the scientific milestones that they would be able to achieve. Finally, we discuss the long-term future of high-contrast stellar interferometry and, in particular, ambitious science cases that would be enabled by space interferometry (e.g., LIFE, space-PFI) and large-scale ground-based projects (PFI).

Keywords: high angular resolution imaging, interferometry, nulling, VLTI, LBTI, KIN, PFN, PFI, LIFE, Hi-5, BIFROST, GRAVITY, ASGARD, planet formation, protoplanetary disks, extrasolar planets, exoplanets, exozodiacal disks

\section{INTRODUCTION}

Direct imaging is a powerful and historically important observing technique in astronomy. From Galileo's lens to modern telescopes, scientific progress and discoveries have been guided by the development of imaging instruments with constantly improving angular resolution, sensitivity, and dynamic range (or contrast). With optical stellar interferometry, there is a routine solution to achieve very high angular resolution but classical visibility or closure phase measurements currently hardly compete with single-dish imaging instruments in terms of contrast. Current classical modal-filtered interferometric instruments such as VLTI/PIONIER, CHARA/FLUOR, and CHARA/MIRC can achieve contrast levels down to $\sim 10^{-3}$ within the diffraction limit of the individual telescopes $(\lambda / \mathrm{D})$ and down to a few milli-arcseconds (mas) using precise visibility and closure phase measurements. ${ }^{1-3}$ This parameter space, only accessible with stellar interferometers, enabled significant scientific results on young stellar objects, bright mature planetary systems, binary companions, and stellar physics. ${ }^{4}$ However, to access the planetary regime, better contrasts are required (see Figure 1).

One of the main challenges to achieve high-contrast observations is to accurately remove the overwhelmingly dominant flux of the host star from the scientific signal, similar to coronagraphy in single-pupil direct imaging. Over the last twenty years, a series of nulling interferometers ${ }^{5}$ have been deployed on state-of-the-art facilities, both across single telescopes and as separate aperture interferometers. These include the BracewelL Infrared Nulling Cryostat ${ }^{6}$ the Keck Interferometer Nuller, ${ }^{7}$ the Palomar Fiber Nuller, ${ }^{8,9}$ the Large Binocular Telescope Interferometer,${ }^{10}$ and DRAGONFLY/GLINT on Subaru/SCExAO. ${ }^{11}$ Considering the most recent three instruments (i.e., GLINT, PFN, and LBTI), the use of nulling interferometry allowed to gain one order of magnitude on the final post-processed contrast levels down to $\sim 10^{-4}$. This can be explained theoretically by the fact that error terms linear in phase and/or amplitude are present at both peak and quadrature, but all linear error terms vanish at null, leaving only smaller quadratic error terms. ${ }^{9}$ The high null depth accuracies obtained with nulling interferometers were also made possible thanks to a combination of factors: the ability to use single-mode fibers (PFN) or integrated optics (GLINT), the use of the telescope's extreme adaptive optics system as a cross-aperture fringe tracker, and the introduction of a significantly improved technique for null-depth measurement, i.e., nulling self calibration. ${ }^{8,12}$ Much was learned about instrumental limitations with the scientific exploitation of these instruments. High-sensitivity mid-IR instruments such as the LBTI is mostly limited by the high thermal background radiation and the excess low frequency noise associated with the detector. At shorter wavelength, where the thermal background is less of an issue, the main limitations are related to high-frequency phase fluctuations and polarization errors. These limitations currently make state-of-the-art nulling interferometer operate one to two orders of magnitudes above the fundamental photon noise limit. ${ }^{13,14}$

Recently, new data acquisition techniques ${ }^{15-18}$ have been proposed in order to better calibrate the instruments and to reach their fundamental photon noise limit. In parallel, a breakthrough has been achieved recently with 

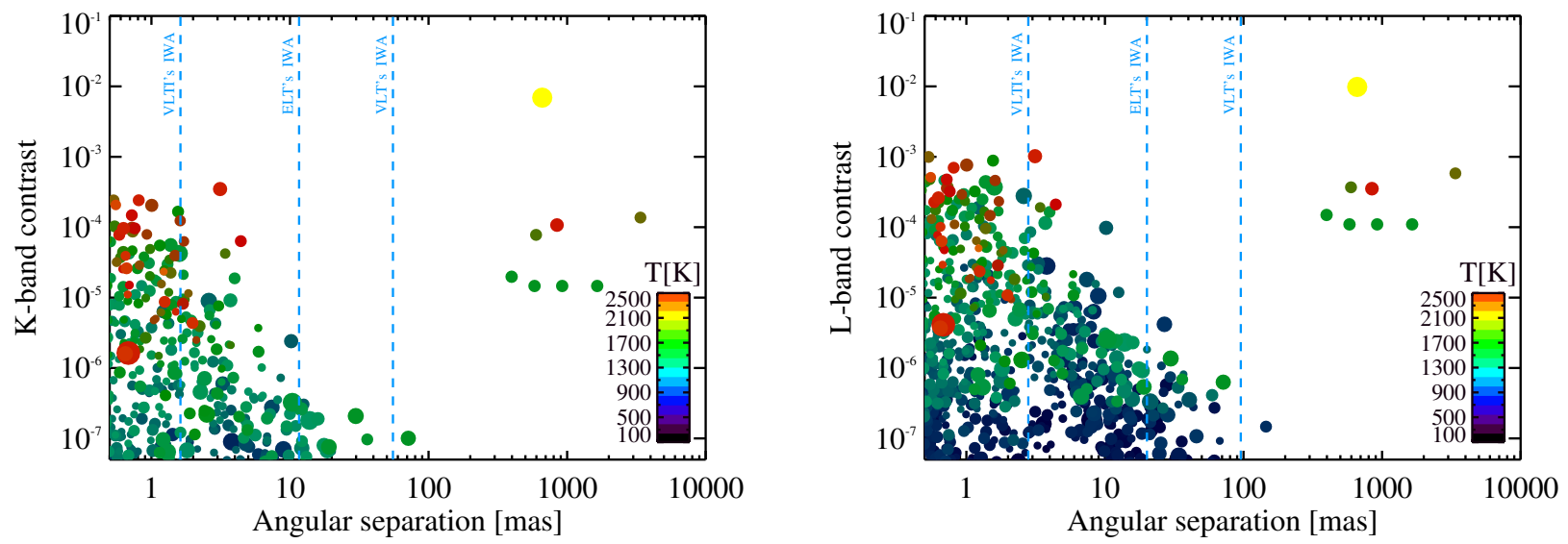

Figure 1. K-band (left) and L-band (right) contrast on known exoplanets vs angular separation (exoplanet data downloaded from NASA exoplanet archiv on November 23rd, 2020). The inner working angle of the VLT/UT, ELT, and VLTI are shown by the dashed vertical lines.

VLTI/GRAVITY demonstrating contrast levels down to $10^{-5}$ at a few $\lambda / \mathrm{D}$ leading to the first direct observation of an exoplanet with long-baseline interferometry ${ }^{19}$ and the first direct observation of an exoplanet discovered by radial velocity by the same team. ${ }^{20}$ These measurements provided record-breaking precision on the astrometry (and hence mass) and spectrum of any directly imaged planet to date. These scientific achievements and contrast levels were enabled by the extreme stability of the GRAVITY instrument and maturity of the VLTI infrastructure. Off-axis observations also enable to get rid of most of the stellar light, similar to what a nulling combination would do. One of the main technological challenges today is to enable this level of contrast for on-axis scientific observations by nulling the host star.

In this paper, we review first the scientific results of high-contrast optical interferometry in Section 2. In Section 3, we present the scientific prospects with existing ground-based instruments (currently being upgraded) and with new visitor instruments currently being investigated. Ambitious science goals enabled by space interferometry are presented in Section 4. We conclude this review of high-contrast optical stellar interferometry in Section 5 .

\section{SCIENCE RESULTS}

\subsection{Exozodiacal dust}

Exozodiacal disks (a.k.a. exozodis) are the extrasolar counterpart of the zodiacal dust found in the solar system. They are both a key to understanding the evolution of planetary systems ${ }^{21}$ and a source of noise for the direct detection of Earth-like exoplanets. ${ }^{22-24}$ Exozodiacal dust emits primarily in the near-infrared to mid-infrared where it is outshone by the host star. Due to the small angular scales involved ( $1 \mathrm{AU}$ at $10 \mathrm{pc}$ corresponds to $0.1 \mathrm{arcsec}$ ), the angular resolution required to spatially disentangle the dust from the stellar emission currently requires the use of interferometry. Thus, exozodis have so far mostly been observed at the CHARA array ${ }^{1,25-27}$ and the $\mathrm{VLTI}^{28-30}$ in the near-infrared, and at $\mathrm{KIN}^{31,32}$ and the $\mathrm{LBTI}^{33-35}$ in the mid-infrared . These observations reached contrasts of a few $10^{-4}$ to a few $10^{-3}$, leading to vital statistical insights into the occurrence rates of exozodis as a function of other properties of the systems such as the presence of cold, Kuiper belt-like dust disks or stellar age and spectral type. Follow-up observations with VLTI/PIONIER, VLTI/GRAVITY, and the PFN of the most interesting systems are now being analysed. In particular, the observations obtained with the PFN, which was decommissioned in 2015 , show no dust detected at the $\sim 0.3 \%$ null upper limit level among the systems with hot dust previously detected by CHARA/FLUOR. ${ }^{26}$ This points to dust too close in for the PFN to resolve with its 3.2-m baseline (Mennesson et al. in prep). For warm dust, the LBTI has completed its core mission in summer 2018 by successfully finishing the Hunt for Observable Signatures of Terrestrial planetary 
Systems (HOSTS) survey. ${ }^{34,35}$ The survey used the instrument's $N$ band nulling interferometric capabilities to search a total of 38 stars for habitable zone (HZ) dust, so-called exozodiacal dust. With a detection rate of $26 \%$ and a sensitivity to dust levels only a few times the Solar system level for the most suitable stars, the LBTI is now able to study common exozodiacal dust systems. The results have shown that there is a clear connection between cold debris disk dust in a system and its HZ dust, but recent work based on the detections has shown that the naive assumption of Poynting-Robertson drag from the outer dust disks to the HZ is insufficient to explain the HZ dust levels. ${ }^{36}$ The statistical results have shown that the dust does generally not prevent exo-Earth imaging with a future space mission such as $\mathrm{HabEx}^{37}$ or LUVOIR ${ }^{38}$ and that even a smaller mission can achieve its imaging goals. However, smaller missions may struggle with the spectroscopic characterization of detected planets. Furthermore, the observations are not yet sensitive enough to provide strong constraints on the HZ dust levels of individual stars in preparation fro exo-Earth imaging. Further observations with the LBTI are now being planned (see Section 3.5) and new instruments are being investigated to cover the Southern sky in the mid-infrared (see Section 3.3).

\subsection{Exoplanet detection and characterization}

The first attempts to detect exoplanets with interferometry used closure phases which are mostly immune to systematic errors from seeing variations. One major limitation of this technique is that the star-planet separation must be resolved while also requiring contrasts of brighter than a few $10^{-4}$, meaning only a few hot Jupiters can be easily accessed (see Figure 1). One helpful effect happens when the star is highly resolved, effectively "boosting" the closure phase signal substantially. ${ }^{39,40}$ Few papers document the many attempts made to use this technique, but one of the earliest was Absil et al., ${ }^{41}$ who set a $5 \times 10^{-3}$ limit for new companions close to $\beta$ Pic using VLTI/AMBER. The first contrast limit better than $10^{-3}$ was made by Zhao et al. $(2011)^{2}$ for upsilon Andromeda B, but with no detection. New attempts with CHARA/MIRCX and VLTI/GRAVITY are underway. New combiners that measure closure phases using nulled outputs of pairwise combiners could dramatically improve the signal-to-noise of this technique ${ }^{15,16,42}$ but this still requires lab development and on-sky testing. Note that if phase referencing or differential phase approaches could work, then more objects could be done since photocenter shifts caused by planets are linear with angular resolution, unlike closure phases which disappear when separation is marginally-resolved. ${ }^{43}$

Recently, a breakthrough has been achieved with VLTI/GRAVITY demonstrating contrast levels down to $10^{-5}$ at a few $\lambda / \mathrm{D}$ leading to the first direct observation of an exoplanet with long-baseline interferometry ${ }^{19}$ and the first direct observation of an exoplanet discovered by radial velocity by the same team. ${ }^{20}$ These measurements provided record-breaking precision on the astrometry (and hence mass) and spectrum of any directly imaged planet to date. These scientific achievements and contrast levels were enabled by the extreme stability of the GRAVITY instrument and maturity of the VLTI infrastructure. Off-axis observations also enable to get rid of most of the stellar light, similar to what a nulling combination would do. Future upgrades of GRAVITY with the GRAVITY+ project will further improve the exoplanet characterization capability of the VLTI.

\section{SCIENCE PROSPECTS FROM THE GROUND}

\subsection{BIFROST: J-band interferometry and high spectral resolution}

The Beam-combination Instrument for studying the Formation and pRoperties of Stars and planeTary systems (BIFROST) is a possible visitor instrument for the VLTI as part of a suite of up to three instruments, together with an L-band nuller (Hi-5/VIKING) and a second-generation fringe tracker (Heimdallr). The three instruments share a common low-order adaptive optics systems to optimise light injection and aim to adopt a shared communication protocol. For the optical design of BIFROST both an all-in-one coaxial beam combination scheme ${ }^{44-46}$ and an integrated optics design ${ }^{47}$ are considered. In order to enable long integration times on the high-spectral resolution arm, we will utilize fringe tracking through the recently-commissioned GRA4MAT mode or the planned second-generation fringe tracker. The goal of the BIFROST instrument concept is to open the short-wavelength window for the VLT Interferometer and to enable interferometry at spectral resolution up to $\lambda / \Delta \lambda=25000 .{ }^{48}$ In the context of high-contrast stellar interferometry, these characteristics will enable several advancements. Many object classes and astrophysical phenomena exhibit more favourable contrast in spectral line emission than in the continuum. One prominent example is planet formation, where the forming 
protoplanets are still embedded in optically thick disks and asymmetric disk dust structures dilute the planet emission and can result in false-positive detections. In these complex environments, accretion-tracing line emission might provide a powerful diagnostic for detecting young planets. ${ }^{49}$ The $\mathrm{Y}$ and J-band $(1-1.3 \mu \mathrm{m})$ are in many respect the ideal wavelength range for spectro-interferometric studies, as they contain spectral line tracers that are not available in other wavebands, such as the accretion-tracing HeI line. Also, the Paschen $\beta$ and $\gamma$ hydrogen recombination lines have higher equivalent width than the commonly-used hydrogen lines accessible at longer wavelengths (Brackett series, etc.), enabling observations at higher signal-to-noise. Furthermore, the high spectral resolution will allow detailed characterisation of the kinematics of the gas that is accreted or ejected from the system.

Pushing towards shorter wavelengths will allow us to achieve a higher angular resolution than other VLTI instruments, enabling the characterisation of companions down to sub-milliarcsecond separation. Also, the GRAVITY instrument has demonstrated that off-axis interferometry can combine the star-light suppression provided by adaptive optics with star-light suppression from interferometry, where photons from an off-axis planet are separated in Fourier phase from stellar photons. ${ }^{50}$ The GRAVITY+ project foresees the installation of improved adaptive optics systems on the UTs, which should provide good Strehl into the J-band, pushing the inner working angle for off-axis interferometry down to $\lambda / D=25$ mas. Applying this technique alongside the GRAVITY + K-band combiner to the J-band will enable high-contrast characterisation of close-in companions and provide access to the rich molecular spectral features that are accessible in this complementary waveband.

\subsection{GLINT: H-band nulling interferometry}

The Guided-Light Interferometric Nulling Technology (GLINT) instrument is a multi-baseline multi-wavelength interferometric nuller, ${ }^{11}$ deployed within the Subaru Coronagraphic Extreme Adaptive Optics (SCExAO) system. Its goal is to pave the way to the development of a science-ready nuller aiming to detect and image exoplanets and study planetary formation. Light in the H-band from the telescope is fragmented in four apertures by an aperture mask in the pupil plane, with individual beams aligned and injected into an integrated-photonic chip by a computer-controlled segmented mirror and a lenslet array. The chip contains waveguides, splitters and couplers to make the light interfere. The outputs after optical processing within the chip consist of a null and an antinull output for every pair of input beams separately: detectors on these waveguides measure the intensities of the dark and bright fringe respectively. The chip also delivers one photometric output for every input beam, enabling continuous monitoring of input intensities and so to correct for instantaneous imbalances in the arms of the interferometer in post-processing. After the chip, the light is dispersed by a prism of spectral resolution of 160 then projected onto the detector. We characterised the instrument and reached a null depth precision of $10^{-4}$ and successfully measured the diameters of $\alpha$ Boo and $\delta$ Vir which sizes are respectively 20 and 10 mas, i.e. below half and below a fifth of the formal diffraction limit of the instrument of 50 mas (Martinod et al., submitted). Next steps are the detection of a companion in a binary system. The exploitation of GLINT shows that atmospheric turbulence, particularly low-order aberrations like low-wind effect, limits the maximum contrast reachable. So it leads to the development of a photonic-component able to correct low-order aberrations and to do fringe tracking while performing nulling.

\subsection{Hi-5: L-band nulling interferometry}

Hi-5 is a high-contrast L-band nulling interferometric instrument for the visitor focus of the VLTI. ${ }^{51}$ By leveraging its state-of-the-art infrastructure, long baselines, and strategic position in the Southern hemisphere, a dedicated high-contrast VLTI instrument will be able to carry out several exoplanet programmes to study young Jupiterlike exoplanets at the most relevant angular separations (i.e., close to the snow line) and better understand how planets form and evolve. First, with a contrast of at least $10^{-5}$ and an inner working angle approximately $\sim 200$ (resp. 20) times better than current 10-m class single-dish telescopes (resp. ELTs), the VLTI would be able to measure the L-band spectra of approximately 25 known exoplanets discovered by radial velocity and currently inaccessible with direct imaging or transit instruments, possibly doubling the number of exoplanet characterised by direct imaging. ${ }^{52}$ In particular, the high-angular resolution provided by the VLTI will give access to the regions of planetary systems located within the snow lines where there is strong evidence for a break in the exoplanet distribution. ${ }^{53}$ The thermal near-infrared is also particularly rich in molecular features, ${ }^{54}$ including $\mathrm{H}_{2} \mathrm{O}$, methane $\left(\mathrm{CH}_{4}\right)$, carbon dioxide $\left(\mathrm{CO}_{2}\right)$, acetylene $\left(\mathrm{C}_{2} \mathrm{H}_{2}\right)$, and hydrogen cyanide $(\mathrm{HCN})$, which will enable 
the detailed characterisation of the chemical composition of the observed exoplanets. Second, another science goal of Hi- 5 is to perform a dedicated survey of nearby young stellar moving groups to search for new giant planets at angular distances inaccessible by current instruments and future ELTs. Approximately 280 young $(<50 \mathrm{Myr})$ and relatively bright $(\mathrm{K}<10)$ stars can be observed from Paranal. ${ }^{52}$ Thanks to its high angular resolution, the VLTI would achieve a higher detection probability than that of instruments installed on 8-m class telescopes and the ELT. ${ }^{16}$ A third important science goal of Hi-5 is the detection and characterization of exozodiacal dust. One of the main challenges at the moment is linking the near-infrared and the mid-infrared detections, which critically constrain the systems' architectures and the properties and origin of the dust. However, so far no connection between the detections in the two wavelength ranges has been found. A new VLTI instrument operating in the thermal near-infrared will be an ideal tool to trace the spectral energy distributions of near-infrared detected exozodis toward longer wavelengths and of mid-infrared detected exozodis toward shorter wavelengths in order to connect the two and to understand non-detections in one wavelength range in the light of detections in the other. Moreover, no sensitive interferometric instrument operating in the thermal near-infrared is available in the Southern hemisphere so far. VLTI/MATISSE is not designed for high-contrast observations and will be limited to the characterisation of the brightest systems already detected in the near-infrared. ${ }^{55}$ With Hi-5, it will be possible to carry out the first large survey of habitable zone dust in the Southern hemisphere.

The Hi-5 project received fundings from OPTICON and was recently funded by the European Research Council (2020-2025) with the main goal to bring the instrument at the visitor focus of the VLTI by the end of 2023. In the long term, Hi-5 will be a cornerstone in the roadmap leading to the characterisation of terrestrial exoplanets and the search for life beyond Earth (see Section 4.2).

\subsection{GRAVITY+}

GRAVITY + is an upgrade of the GRAVITY instrument. ${ }^{56}$ The on-going upgrade will provide an extreme Adaptive Optics to replace the 20-year old system that feeds the instrument (and with which GRAVITY has already shown unprecedented performances at short separations). This is a critical ingredient to increase the contrast of GRAVITY. For the first time the need to optimize a dedicated high-contrast mode is part of GRAVITY, and should pay by increased contrast ratio.

One of the science goal of GRAVITY+ is to prepare the instrument for the incoming GAIA releases. For stars at $20 \mathrm{pc}$ from the Sun and a nominal 5-year mission, Gaia's peak sensitivity corresponds to planets at 100 mas or $2 \mathrm{AU}$ from their host star. This is just too small for measuring their infrared flux with classical imaging. But it falls well in the range of interferometric imaging. At a separation of 130 mas, $\beta$ Pictoris $\mathrm{c}$ is actually a prototypical example (see Figure 2). We expect a few of them within the current contrast limit of GRAVITY (many others at larger separations will be observable with classical imaging, but they are much less challenging for the formation theories).

\subsection{LBTI: LM-band Fizeau interferometry and N-band nulling interferometry}

A description of the LBTI has been presented by Hinz et al. (2016) ${ }^{57}$ and an update will be presented in this series. ${ }^{58}$ Since the completion of the HOSTS and LEECH surveys, ${ }^{59}$ the LBTI team has consolidated funding and long-term support for the instrument and worked on a long-term plan for the instrument. On the technical side, the team has focused on maturing the adaptive optics (AO) assisted direct imaging modes, including the Arizona Lenslet for Exoplanet Spectroscopy (ALES) thermal infrared integral field spectroscopy mode ${ }^{60}$ and a sensitive high-contrast imaging mode with the NOMIC ${ }^{61}$ mid-infrared camera (PI: K. Wagner). In addition, the team has studied a range of upgrades to enhance the instrument's nulling and Fizeau interferometric capabilities and on making the Fizeau mode more routine. On nulling interferometry, the team has produced a detailed study of the current instrument performance and of realistic improvements. ${ }^{62}$ It was shown that the two dominant sources of uncertainty are low frequency telescope and instrument vibrations and detector Excess Low Frequency Noise (ELFN). The mitigation of vibrations has been made a priority by the observatory and is in progress. The team is also testing a new H1RG detector with a sensitivity up to a wavelength of $13 \mu \mathrm{m}^{63}$ that promises to be ELFN free. It has been shown that eliminating the ELFN and realistic reduction of vibrations can improve the sensitivity by a factor of three. This would make a strong case for a renewed HOSTS survey. In addition, the LBTI team has been awarded NASA/XRP funding (PI: S. Ertel) for the characterization of previously detected 


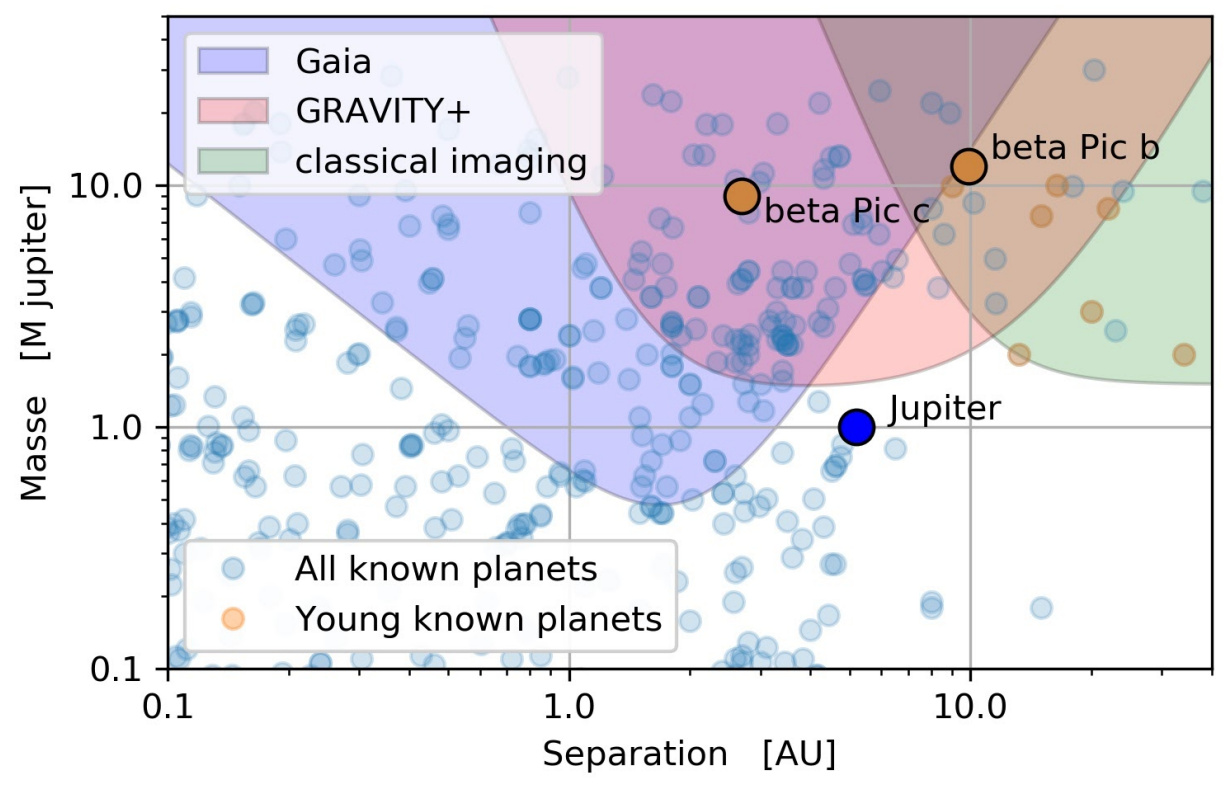

Figure 2. Sensitivity region of Gaia, classical imaging and interferometric imaging, overlaid to known planets from the NASA database. Because of observational and astrophysical biases, most known young planets are at $>10$ AU (brown in this picture). Thanks to its exhaustive approach, Gaia will detect many more of these young planets at $<10$ AU. We know this population of young planets should exist because we already detect its older counterpart (blue in this picture). GRAVITY + is unique to characterise the intrinsic infrared flux and thus the formation entropy of these young planets.

exozodiacal dust. ${ }^{64}$ The planned observations in two filters across the $N$ band and with a wide position angle coverage of the interferometric baseline will result in a detailed characterization of the radial and azimuthal structure and the spectral shape of the dust emission.

The LBTI's Fizeau imaging interferometry mode has been rarely used due to the instrument team's previous science focus on nulling interferometry. It is currently being matured for routine operations. ${ }^{65,66}$ Currently, one of the main limitations for for precision, high-fidelity, and high-contrast interferometric imaging with a 23-m equivalent aperture is the limited sensitivity of the PhaseCam fringe $\operatorname{tracker}^{67}$ (limiting magnitude $K \sim$ 4.5). PhaseCam is currently equipped with a PICNIC detector and an upgrade to a SAPHIRA detector is in preparation (funded, PI: J. Stone). This upgrade together with other minor improvements is expected to result in a new limiting magnitude of $K \sim 10$. Together with the recently completed Single-conjugated adaptive Optics Upgrade for the LBT (SOUL) ${ }^{68}$ with a high-performance limiting magnitude of $R \sim 12.5$, this will open up a significant number of young stellar objects in nearby star forming regions for high contrast 23-m resolution $L$ and $M$ band imaging (Fig. 3). The Fizeau mode will also be used for general astronomical observations such as the high-contrast imaging search for planets around nearby, bright stars, Solar system science, extra-galactic astronomy, and the study of evolved stars. The mode can be further extended to be combined with coronagraphy and ALES integral field spectroscopy. On the longer term, there is the possibility to extend the Fizeau imaging mode toward shorter wavelengths, including potentially visible wavelengths. ${ }^{69}$

\subsection{PFI: Planet Formation Imager}

$\mathrm{PFI}^{70}$ is currently a science-driven, international initiative to develop the roadmap for a future ground-based facility that will be optimised to image planet-forming disks on the spatial scale where the protoplanets are assembled, which is the Hill sphere of the forming planets. The goal of PFI will be to detect and characterise protoplanets during their first $\sim 100$ million years and trace how the planet population changes due to migration processes, unveiling the processes that determine the final architecture of exoplanetary systems. With $\sim 20$ telescope elements and baselines of $\sim 3 \mathrm{~km}$, the PFI concept is optimised for imaging complex scenes at thermal near-infrared and mid-infrared wavelengths $(3-12 \mu \mathrm{m})$ and at 0.1 milliarcsecond resolution. 


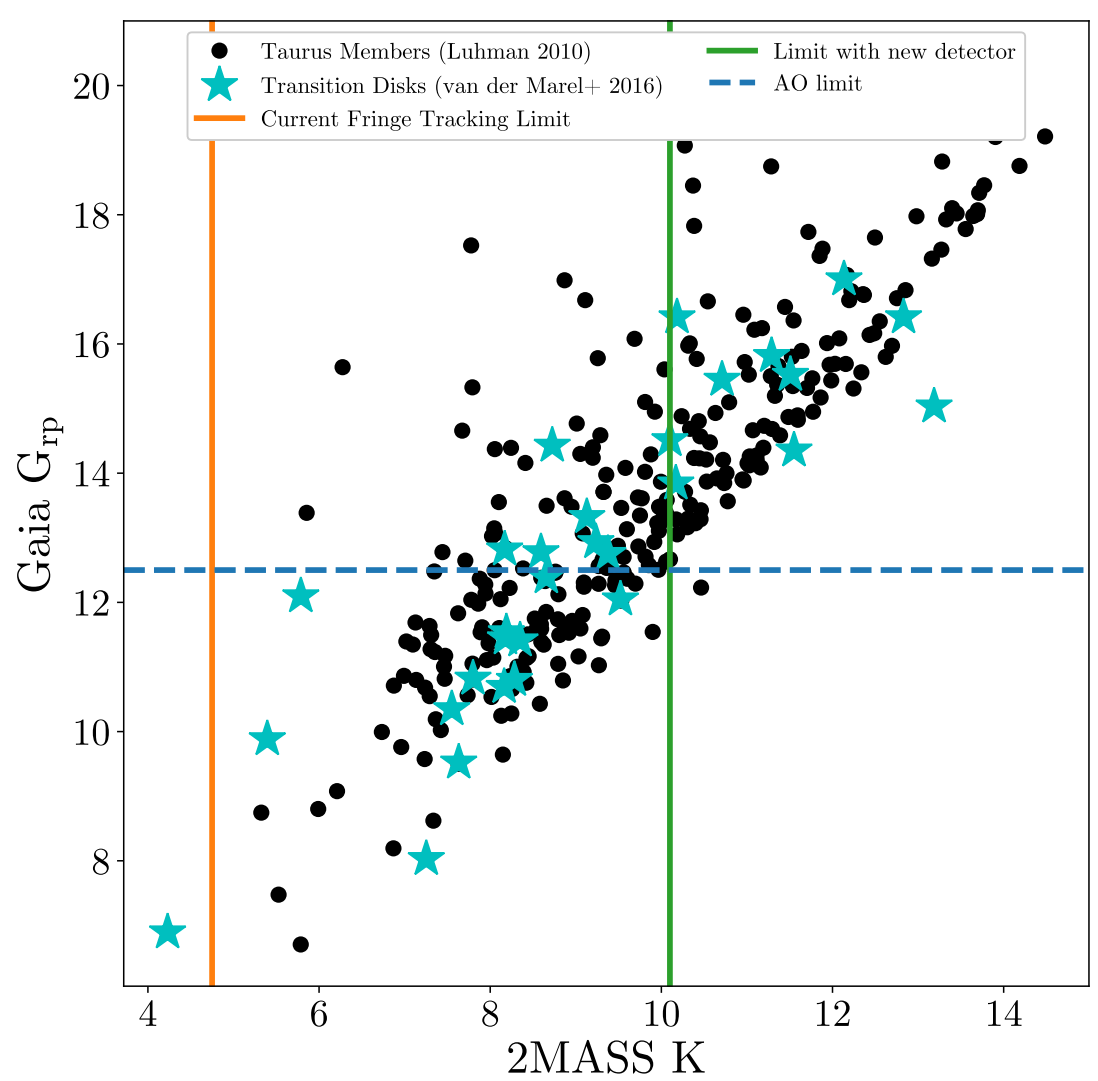

Figure 3. Taurus young stellar objects and transition disks that can be imaged with the current and future fringe tracking capabilities of the LBTI.

\section{SCIENCE PROSPECTS FROM SPACE}

\subsection{Demonstration missions on small platforms}

The path towards space-based interferometry is regularly discussed in the community and these discussions often involve the need for precursor missions. ${ }^{71,72}$ Concepts of small-scale space-based infrared nulling interferometers were seriously considered in the 2000's both in Europe and in the US: the Fourier-Kelvin Stellar Interferometer ${ }^{73,74}$ and Pegase. ${ }^{75}$ More recently, concepts for interferometric platforms have been proposed, such as FIRST-S ${ }^{76}$ a $3 \mathrm{U}$ CubeSat with a Lithium Niobate nulling combiner. The technical challenges of the project are: star tracking, beam combination, and nulling capabilities. The optical baseline of the interferometer would be $30 \mathrm{~cm}$, giving a $2.2 \mathrm{AU}$ spatial resolution at distance of $10 \mathrm{pc}$. The scientific objective of this mission would be to study the visible emission of exozodiacal light in the habitable zone around the closest stars. Another project to demonstrate a linear formation-flying astronomical interferometer in low Earth orbit is also currently under study. ${ }^{77}$ To detect exoplanets, larger platforms are required as recently studied by Dandumont et al. (2020). ${ }^{78}$ Figure 4 shows the exoplanet yield analysis assuming the Kepler occurrence rate and assuming four different space-based nulling interferometer concepts (2-aperture fibered Bracewell). Two CubeSats (baseline: $0.5 \mathrm{~m} / 1.0 \mathrm{~m}$ and apertures: $0.08 \mathrm{~m}$ ), an ESA PROBA-like mission (baseline: $5.0 \mathrm{~m}$ and apertures: $0.25 \mathrm{~m}$ ), and the FKSI concept (baseline: $12.5 \mathrm{~m}$ and apertures: $0.5 \mathrm{~m}$ ) were considered. They show that, even without platform stability constraints, CubeSats can hardly detect giant exoplanets. A PROBA-like mission could detect more than 120 exoplanets and a more ambitious mission such as FKSI could detect 250 exoplanets. One of their conclusions is that small platforms are well suited to test and validate critical technological components needed for a larger mission and perform scientific observations (see Dandumont et al. in this series for more information). Further investigations are however required to estimate the impact of instrumental noise. 


\section{CubeSat 6U}

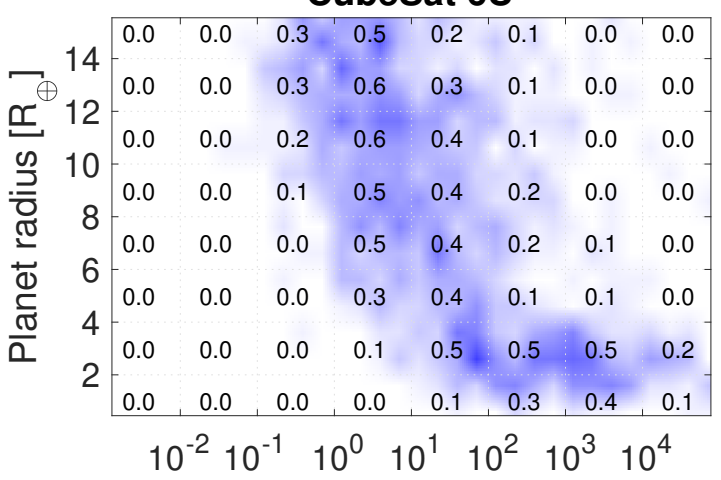

Stellar insolation [solar constant]

PROBA-size

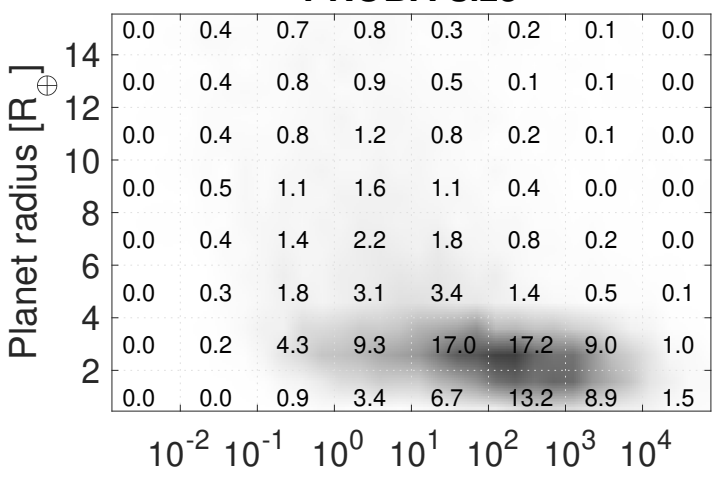

Stellar insolation [solar constant]

\section{CubeSat 12U}

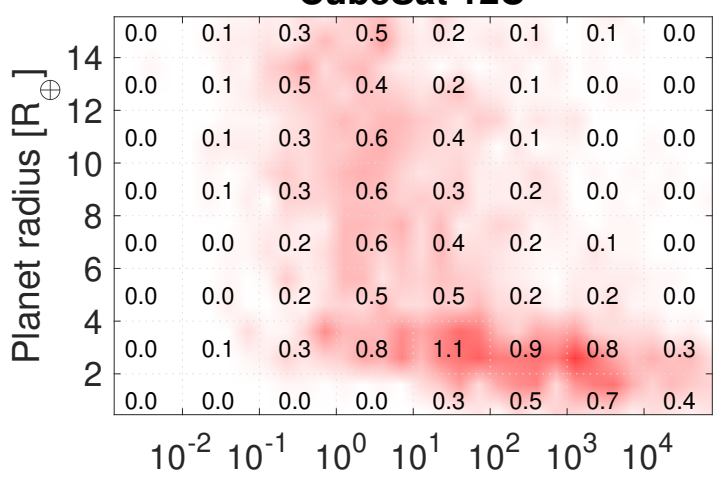

Stellar insolation [solar constant]

FKSI concept

\begin{tabular}{|c|c|c|c|c|c|c|c|c|}
\hline \multirow{2}{*}{$\tau^{14}$} & 0.0 & 0.3 & 0.7 & 0.9 & 0.3 & 0.2 & 0.1 & 0.0 \\
\hline & 0.0 & 0.3 & 0.8 & 1.0 & 0.5 & 0.1 & 0.1 & 0.0 \\
\hline & 0.0 & 0.3 & 0.7 & 1.5 & 0.8 & 0.2 & 0.1 & 0.0 \\
\hline & 0.0 & 0.4 & 1.0 & 2.3 & 1.1 & 0.4 & 0.0 & 0.0 \\
\hline & 0.0 & 0.3 & 1.2 & 3.3 & 2.0 & 0.8 & 0.2 & 0.0 \\
\hline & 0.0 & 0.2 & 1.6 & 5.8 & 4.2 & 1.4 & 0.5 & 0.1 \\
\hline & 0.0 & 0.1 & 4.3 & 26.2 & 38.8 & 21.2 & 9.3 & 1.0 \\
\hline & 0.0 & 0.0 & 1.1 & 15.0 & 59.7 & 50.1 & 20.5 & 2.2 \\
\hline
\end{tabular}

Figure 4. Mean number of exoplanet detection as a function of stellar insolation and planet radius assuming the Kepler occurrence rate and four different instrument configurations: ${ }^{78}$ a $6 \mathrm{U}$ cubesat with a baseline of $0.5 \mathrm{~m}$ and two apertures of $8 \mathrm{~cm}$ in diameter, a $12 \mathrm{U}$ cubesat with a baseline of $1 \mathrm{~m}$ and two apertures of $8 \mathrm{~cm}$ in diameter, a PROBA-size platform with a baseline of $5 \mathrm{~m}$ and two apertures of $25 \mathrm{~cm}$ in diameter, and a FKSI concept with a baseline of $12.5 \mathrm{~m}$ and two apertures of $50 \mathrm{~cm}$ in diameter. The exoplanet yield is computed based on pure photometric assumptions and does not assume instrumental noise related to tip/tilt and OPD errors.

\subsection{LIFE: the Large Interferometer For Exoplanets}

LIFE is an initiative* to develop the science, technology and a roadmap for an ambitious space mission that will allow humankind to detect dozens of warm, terrestrial exoplanets and hundreds of exoplanets overall at mid-infrared (MIR) wavelengths. ${ }^{79,80}$ For most of the detected exoplanets direct estimates of their effective temperature and radius will be available, and a for a significant subset the atmospheric composition will be investigated including the search for potential bio-signatures. ${ }^{80-82}$ Characterizing exoplanet atmospheres using their thermal emission at MIR wavelengths - compared to studies at optical/near-infrared wavelength looking at planets in reflected light — offers the possibility to study a broader set of molecular features ${ }^{83}$ and get a better understanding of the atmospheric structure. ${ }^{84}$ Hence, in particular for questions related to the habitability of exoplanets, a mission like LIFE offers unprecedented scientific potential.

The current baseline design of LIFE features a 4-aperture interferometer array with a $6: 1$ baseline ratio to reduce the impact of instability noise. ${ }^{85,86}$ A beam combiner spacecraft is located at the center of the array. The size of the individual apertures is currently under study, but based on detection yield simulations including all relevant astrophysical noise sources (see, Figure 5, diameters of 2-3.5 m are under consideration. The aperture size is primarily driven by the number of detectable planets and the time-on-target required for in-depth atmospheric

*www.life-space-mission.com 


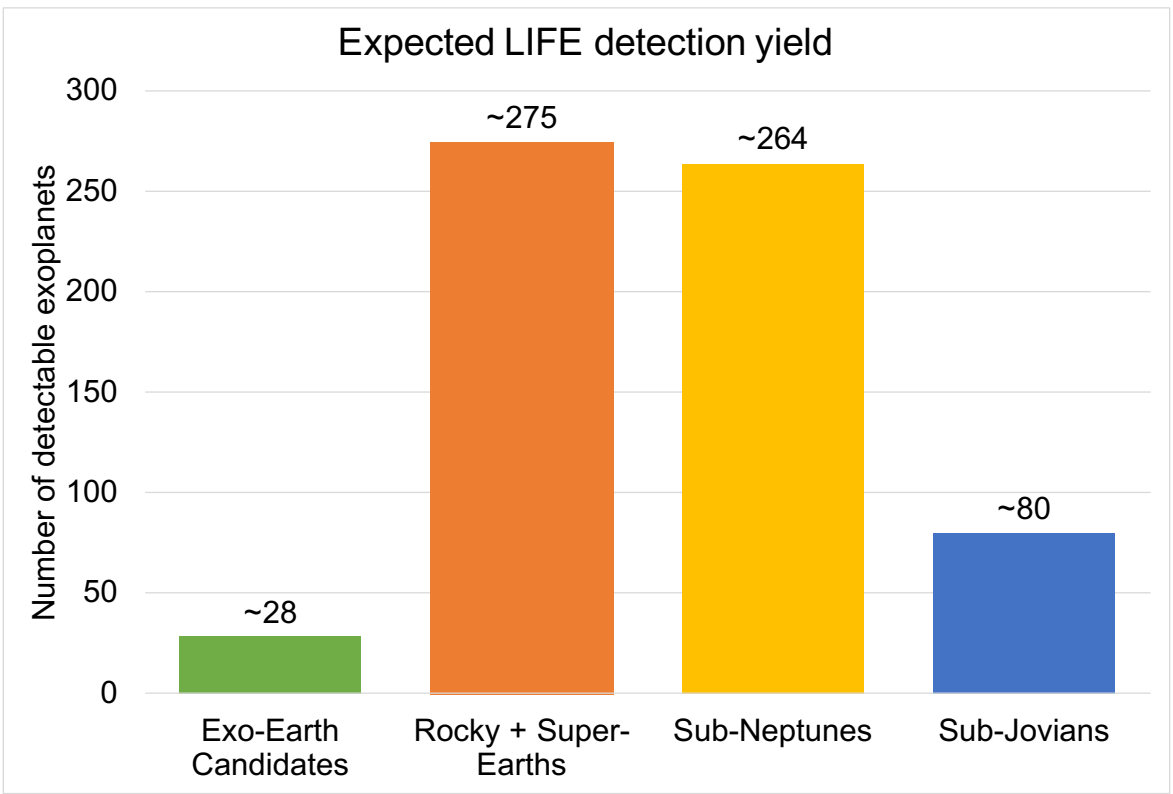

Figure 5. Expected LIFE exoplanet detection yield for a 2.5 year search phase and assuming an aperture size of $\mathrm{D}=3.5 \mathrm{~m}$ (cf. Quanz et al., submitted). The exoplanet classification scheme used here is the same as in Kopparapu et al. (2018). ${ }^{90}$ The Monte-Carlo approach underlying these results is described in previous studies. ${ }^{80,91}$

characterization. The current wavelength range requirement is $4-18.5 \mu \mathrm{m}$, but additional studies are underway for further verification. A spectral resolution of at least $R=30$, but better $R=50$, seems required in order to reliably quantify the abundance ratios of main molecular species in the atmosphere of an Earth-twin planet at several pc distance. The minimum mission lifetime is 5-6 years in order to have sufficient time for both a dedicated search phase, to identify the most interesting and promising targets, and a characterization phase for in-depth investigations of a subset of those. LIFE shall be launched to the Earth-Sun L2 point.

One of the crucial next steps to advance the technological readiness of LIFE is related to nulling at MIR wavelengths. While in the context of Darwin and TPF-I the general feasibility of the required null-depth and stability was demonstrated by Martin et al. (2012), ${ }^{87}$ these earlier lab experiments were done at ambient temperatures and with high flux levels. A corresponding experiment, but under cryogenic conditions and with flux levels in-line with those expected from astronomical sources, is underway in the form of the Nulling Interferometric Cryogenic Experiment (NICE) at ETH Zurich (Gheorghe et al., in prep.). A more general overview of the readiness of key technologies for a space mission like LIFE was presented in recent reviews. ${ }^{88,89}$

\section{CONCLUSION}

The field of high-contrast stellar interferometry has made significant progress over the past decade with recordbreaking high-contrast observations with nulling interferometric instruments and the first detection and characterization of exoplanets with long-baseline interferometry. Current state-of-the-art contrasts amount to $\sim 10^{-4}$ within the diffraction limit of individual telescopes using nulling interferometry ( $\mathrm{H}, \mathrm{K}$, and $\mathrm{N}$ bands) and to $\sim 10^{-5}$ for off-axis observations using dual-feed interferometry at the VLTI with the GRAVITY instrument (K band). Pushing these high-contrast capabilities to smaller inner working angles is today crucial to make scientific progress in various fields of astrophysics and, in particular, in exoplanet science. While current instruments are currently being upgraded to improve their sensitivity or contrast limits (e.g., GLINT, LBTI, MIRCX, GRAVITY + ), this can also be achieved by developing new instruments operating at shorter wavelength (e.g., BIFROST at $\mathrm{J}$ band) or by building the first nulling instrument for the VLTI (Hi-5/VIKING at L band). New ideas and technology solutions have also emerged to improve the contrast of long-baseline interferometers such as combining nulling and closure phase, kernel nulling, advanced fringe tracking, and high-dispersion interferometry. These techniques now need to be tested and validated on sky. In the long term, these developments will serve as 
a key technology demonstrator for future major interferometric instruments such as PFI and LIFE for the most ambitious science cases.

\section{ACKNOWLEDGMENTS}

S.K. and D.D. acknowledge funding from the European Research Council (grant agreement No. 639889 and 866070, respectively).

\section{REFERENCES}

[1] Absil, O., di Folco, E., Mérand, A., Augereau, J.-C., Coudé du Foresto, V., Aufdenberg, J. P., Kervella, P., Ridgway, S. T., Berger, D. H., ten Brummelaar, T. A., Sturmann, J., Sturmann, L., Turner, N. H., and McAlister, H. A., "Circumstellar material in the Vega inner system revealed by CHARA/FLUOR," Astronomy and Astrophysics 452, 237-244 (June 2006).

[2] Zhao, M., Monnier, J. D., Che, X., Pedretti, E., Thureau, N., Schaefer, G., ten Brummelaar, T., Mérand, A., Ridgway, S. T., McAlister, H., Turner, N., Sturmann, J., Sturmann, L., Goldfinger, P. J., and Farrington, C., "Toward Direct Detection of Hot Jupiters with Precision Closure Phase: Calibration Studies and First Results from the CHARA Array," Publications of the Astronomical Society of the Pacific 123, 964-975 (Aug. 2011).

[3] Marion, L., Absil, O., Ertel, S., Bouquin, J.-B. L., Augereau, J.-C., Blind, N., Defrère, D., Lebreton, J., and Milli, J., "Searching for faint companions with VLTI/PIONIER - II. 92 main sequence stars from the Exozodi survey," Astronomy 83 Astrophysics 570, A127 (Oct. 2014). Publisher: EDP Sciences.

[4] Defrère, D., Aerts, C., Kishimoto, M., and Léna, P., "A recent history of science cases for optical interferometry," Experimental Astronomy 46, 389-399 (Dec. 2018).

[5] Bracewell, R. N., "Detecting nonsolar planets by spinning infrared interferometer," Nature 274, 780 (Aug. 1978).

[6] Hinz, P. M., Angel, J. R. P., Hoffmann, W. F., McCarthy, D. W., McGuire, P. C., Cheselka, M., Hora, J. L., and Woolf, N. J., "Imaging circumstellar environments with a nulling interferometer," Nature 395, 251-253 (Sept. 1998). Number: 6699 Publisher: Nature Publishing Group.

[7] Serabyn, E., Mennesson, B., Colavita, M. M., Koresko, C., and Kuchner, M. J., "THE KECK INTERFEROMETER NULLER," The Astrophysical Journal 748, 55 (Mar. 2012).

[8] Mennesson, B., Hanot, C., Serabyn, E., Liewer, K., Martin, S. R., and Mawet, D., "HIGH-CONTRAST STELLAR OBSERVATIONS WITHIN THE DIFFRACTION LIMIT AT THE PALOMAR HALE TELESCOPE," The Astrophysical Journal 743, 178 (Dec. 2011).

[9] Serabyn, E., Mennesson, B., Martin, S., Liewer, K., and Kühn, J., "Nulling at short wavelengths: theoretical performance constraints and a demonstration of faint companion detection inside the diffraction limit with a rotating-baseline interferometer," Monthly Notices of the Royal Astronomical Society 489, 1291-1303 (Oct. 2019).

[10] Hinz, P. M., Defrère, D., Skemer, A., Bailey, V., Stone, J., Spalding, E., Vaz, A., Pinna, E., Puglisi, A., Esposito, S., Montoya, M., Downey, E., Leisenring, J., Durney, O., Hoffmann, W., Hill, J., Millan-Gabet, R., Mennesson, B., Danchi, W., Morzinski, K., Grenz, P., Skrutskie, M., and Ertel, S., "Overview of LBTI: a multipurpose facility for high spatial resolution observations," 9907, 990704 (Aug. 2016).

[11] Norris, B. R. M., Cvetojevic, N., Lagadec, T., Jovanovic, N., Gross, S., Arriola, A., Gretzinger, T., Martinod, M.-A., Guyon, O., Lozi, J., Withford, M. J., Lawrence, J. S., and Tuthill, P., "First on-sky demonstration of an integrated-photonic nulling interferometer: the GLINT instrument," Monthly Notices of the Royal Astronomical Society 491, 4180-4193 (Jan. 2020).

[12] Hanot, C., Mennesson, B., Martin, S., Liewer, K., Loya, F., Mawet, D., Riaud, P., Absil, O., and Serabyn, E., "Improving Interferometric Null Depth Measurements using Statistical Distributions: Theory and First Results with the Palomar Fiber Nuller," The Astrophysical Journal 729, 110 (Mar. 2011). 
[13] Colavita, M. M., Serabyn, E., Millan-Gabet, R., Koresko, C. D., Akeson, R. L., Booth, A. J., Mennesson, B. P., Ragland, S. D., Appleby, E. C., Berkey, B. C., Cooper, A., Crawford, S. L., Creech-Eakman, M. J., Dahl, W., Felizardo, C., Garcia-Gathright, J. I., Gathright, J. T., Herstein, J. S., Hovland, E. E., Hrynevych, M. A., Ligon, E. R., Medeiros, D. W., Moore, J. D., Morrison, D., Paine, C. G., Palmer, D. L., Panteleeva, T., Smith, B., Swain, M. R., Smythe, R. F., Summers, K. R., Tsubota, K., Tyau, C., Vasisht, G., Wetherell, E., Wizinowich, P. L., and Woillez, J. M., "Keck Interferometer Nuller Data Reduction and On-Sky Performance," Publications of the Astronomical Society of the Pacific 121, 1120-1138 (Oct. 2009).

[14] Defrère, D., Hinz, P. M., Mennesson, B., Hoffmann, W. F., Millan-Gabet, R., Skemer, A. J., Bailey, V., Danchi, W. C., Downey, E. C., Durney, O., Grenz, P., Hill, J. M., McMahon, T. J., Montoya, M., Spalding, E., Vaz, A., Absil, O., Arbo, P., Bailey, H., Brusa, G., Bryden, G., Esposito, S., Gaspar, A., Haniff, C. A., Kennedy, G. M., Leisenring, J. M., Marion, L., Nowak, M., Pinna, E., Powell, K., Puglisi, A., Rieke, G., Roberge, A., Serabyn, E., Sosa, R., Stapeldfeldt, K., Su, K., Weinberger, A. J., and Wyatt, M. C., "Nulling Data Reduction and On-sky Performance of the Large Binocular Telescope Interferometer," The Astrophysical Journal 824, 66 (June 2016).

[15] Lacour, S., Tuthill, P., Monnier, J. D., Kotani, T., Gauchet, L., and Labeye, P., "A new interferometer architecture combining nulling with phase closure measurements," Monthly Notices of the Royal Astronomical Society 439, 4018-4029 (Apr. 2014).

[16] Martinache, F. and Ireland, M. J., "Kernel-nulling for a robust direct interferometric detection of extrasolar planets," Astronomy $\& 3$ Astrophysics 619, A87 (Nov. 2018). Publisher: EDP Sciences.

[17] Laugier, R., Cvetojevic, N., and Martinache, F., "Kernel nullers for an arbitrary number of apertures," Astronomy $\&$ Astrophysics 642, A202 (Oct. 2020). Publisher: EDP Sciences.

[18] Martinache, F., Ceau, A., Laugier, R., Kammerer, J., N’Diaye, M., Mary, D., Cvetojevic, N., and Lopez, C., "Kernel-phase analysis: Aperture modeling prescriptions that minimize calibration errors," Astronomy \&5 Astrophysics 636, A72 (Apr. 2020).

[19] Lacour, S., Dembet, R., Abuter, R., Fédou, P., Perrin, G., Choquet, E., Pfuhl, O., Eisenhauer, F., Woillez, J., Cassaing, F., Wieprecht, E., Ott, T., Wiezorrek, E., Tristram, K. R. W., Wolff, B., Ramírez, A., Haubois, X., Perraut, K., Straubmeier, C., Brandner, W., and Amorim, A., "The GRAVITY fringe tracker," Astronomy and Astrophysics 624, A99 (Apr. 2019).

[20] Nowak, M., Lacour, S., Lagrange, A.-M., Rubini, P., Wang, J., Stolker, T., Abuter, R., Amorim, A., Asensio-Torres, R., Bauböck, M., Benisty, M., Berger, J. P., Beust, H., Blunt, S., Boccaletti, A., Bonnefoy, M., Bonnet, H., Brandner, W., Cantalloube, F., Charnay, B., Choquet, E., Christiaens, V., Clénet, Y., Coudé Du Foresto, V., Cridland, A., de Zeeuw, P. T., Dembet, R., Dexter, J., Drescher, A., Duvert, G., Eckart, A., Eisenhauer, F., Gao, F., Garcia, P., Garcia Lopez, R., Gardner, T., Gendron, E., Genzel, R., Gillessen, S., Girard, J., Grandjean, A., Haubois, X., Heißel, G., Henning, T., Hinkley, S., Hippler, S., Horrobin, M., Houllé, M., Hubert, Z., Jiménez-Rosales, A., Jocou, L., Kammerer, J., Kervella, P., Keppler, M., Kreidberg, L., Kulikauskas, M., Lapeyrère, V., Le Bouquin, J.-B., Léna, P., Mérand, A., Maire, A.-L., Mollière, P., Monnier, J. D., Mouillet, D., Müller, A., Nasedkin, E., Ott, T., Otten, G., Paumard, T., Paladini, C., Perraut, K., Perrin, G., Pueyo, L., Pfuhl, O., Rameau, J., Rodet, L., Rodríguez-Coira, G., Rousset, G., Scheithauer, S., Shangguan, J., Stadler, J., Straub, O., Straubmeier, C., Sturm, E., Tacconi, L. J., van Dishoeck, E. F., Vigan, A., Vincent, F., von Fellenberg, S. D., Ward-Duong, K., Widmann, F., Wieprecht, E., Wiezorrek, E., Woillez, J., and Gravity Collaboration, "Direct confirmation of the radialvelocity planet beta Pictoris c," Astronomy and Astrophysics 642, L2 (Oct. 2020).

[21] Kral, Q., Krivov, A. V., Defrère, D., van Lieshout, R., Bonsor, A., Augereau, J.-C., Thébault, P., Ertel, S., Lebreton, J., and Absil, O., "Exozodiacal clouds: hot and warm dust around main sequence stars," The Astronomical Review 13, 69-111 (Apr. 2017).

[22] Defrère, D., Stark, C., Cahoy, K., and Beerer, I., "Direct imaging of exoEarths embedded in clumpy debris disks," 8442, 84420M (Sept. 2012).

[23] Roberge, A., Chen, C. H., Millan-Gabet, R., Weinberger, A. J., Hinz, P. M., Stapelfeldt, K. R., Absil, O., Kuchner, M. J., and Bryden, G., "The Exozodiacal Dust Problem for Direct Observations of Exo-Earths," Publications of the Astronomical Society of the Pacific 124, 799 (Aug. 2012). 
[24] Stark, C. C., Schneider, G., Weinberger, A. J., Debes, J. H., Jang-Condell, H., Grady, C. A., Carson, J., Henning, T., Hines, D. C., Hinz, P., Kuchner, M. J., Moro-Martin, A., Perrin, M. D., Tamura, M., Serabyn, G., Silverstone, M. D., Goto, M., and Wisniewski, J. P., "HD 181327 Debris Disk Asymmetries: Signs of a Planet or Geometric Projection Effects?," 223, 303.06 (Jan. 2014).

[25] Absil, O., di Folco, E., Mérand, A., Augereau, J.-C., Coudé du Foresto, V., Defrère, D., Kervella, P., Aufdenberg, J. P., Desort, M., Ehrenreich, D., Lagrange, A.-M., Montagnier, G., Olofsson, J., ten Brummelaar, T. A., McAlister, H. A., Sturmann, J., Sturmann, L., and Turner, N. H., "A near-infrared interferometric survey of debris disc stars. II. CHARA/FLUOR observations of six early-type dwarfs," Astronomy and Astrophysics 487, 1041-1054 (Sept. 2008).

[26] Absil, O., Defrère, D., Coudé du Foresto, V., Di Folco, E., Mérand, A., Augereau, J.-C., Ertel, S., Hanot, C., Kervella, P., Mollier, B., Scott, N., Che, X., Monnier, J. D., Thureau, N., Tuthill, P. G., ten Brummelaar, T. A., McAlister, H. A., Sturmann, J., Sturmann, L., and Turner, N., "A near-infrared interferometric survey of debris-disc stars. III. First statistics based on 42 stars observed with CHARA/FLUOR," Astronomy and Astrophysics 555, A104 (July 2013).

[27] Nuñez, P. D., Scott, N. J., Mennesson, B., Absil, O., Augereau, J.-C., Bryden, G., ten Brummelaar, T., Ertel, S., Coudé du Foresto, V., Ridgway, S. T., Sturmann, J., Sturmann, L., Turner, N. J., and Turner, N. H., "A near-infrared interferometric survey of debris-disc stars: VI. Extending the exozodiacal light survey with CHARA/JouFLU," Astronomy 83 Astrophysics 608, A113 (Dec. 2017).

[28] Defrère, D., Lebreton, J., Le Bouquin, J.-B., Lagrange, A.-M., Absil, O., Augereau, J.-C., Berger, J.P., di Folco, E., Ertel, S., Kluska, J., Montagnier, G., Millan-Gabet, R., Traub, W., and Zins, G., "Hot circumstellar material resolved around beta Pic with VLTI/PIONIER," Astronomy and Astrophysics 546, L9 (Oct. 2012).

[29] Ertel, S., Absil, O., Defrère, D., Le Bouquin, J.-B., Augereau, J.-C., Marion, L., Blind, N., Bonsor, A., Bryden, G., Lebreton, J., and Milli, J., "A near-infrared interferometric survey of debris-disk stars. IV. An unbiased sample of 92 southern stars observed in H band with VLTI/PIONIER," Astronomy and Astrophysics 570, A128 (Oct. 2014).

[30] Ertel, S., Defrère, D., Absil, O., Le Bouquin, J.-B., Augereau, J.-C., Berger, J.-P., Blind, N., Bonsor, A., Lagrange, A.-M., Lebreton, J., Marion, L., Milli, J., and Olofsson, J., "A near-infrared interferometric survey of debris-disc stars. V. PIONIER search for variability," Astronomy and Astrophysics 595, A44 (Oct. 2016).

[31] Millan-Gabet, R., Mennesson, B., Hinz, P., Kuchner, M., and Serabyn, G., "Exozodi Dust Emission Measured with the Keck Interferometer Nuller," 221, 403.05 (Jan. 2013).

[32] Mennesson, B., Millan-Gabet, R., Serabyn, E., Colavita, M. M., Absil, O., Bryden, G., Wyatt, M., Danchi, W., Defrère, D., Doré, O., Hinz, P., Kuchner, M., Ragland, S., Scott, N., Stapelfeldt, K., Traub, W., and Woillez, J., "Constraining the Exozodiacal Luminosity Function of Main-sequence Stars: Complete Results from the Keck Nuller Mid-infrared Surveys," The Astrophysical Journal 797, 119 (Dec. 2014).

[33] Defrère, D., Hinz, P. M., Skemer, A. J., Kennedy, G. M., Bailey, V. P., Hoffmann, W. F., Mennesson, B., Millan-Gabet, R., Danchi, W. C., Absil, O., Arbo, P., Beichman, C., Brusa, G., Bryden, G., Downey, E. C., Durney, O., Esposito, S., Gaspar, A., Grenz, P., Haniff, C., Hill, J. M., Lebreton, J., Leisenring, J. M., Males, J. R., Marion, L., McMahon, T. J., Montoya, M., Morzinski, K. M., Pinna, E., Puglisi, A., Rieke, G., Roberge, A., Serabyn, E., Sosa, R., Stapeldfeldt, K., Su, K., Vaitheeswaran, V., Vaz, A., Weinberger, A. J., and Wyatt, M. C., "First-light LBT Nulling Interferometric Observations: Warm Exozodiacal Dust Resolved within a Few AU of eta Crv," The Astrophysical Journal 799, 42 (Jan. 2015).

[34] Ertel, S., Defrère, D., Hinz, P., Mennesson, B., Kennedy, G. M., Danchi, W. C., Gelino, C., Hill, J. M., Hoffmann, W. F., Rieke, G., Shannon, A., Spalding, E., Stone, J. M., Vaz, A., Weinberger, A. J., Willems, P., Absil, O., Arbo, P., Bailey, V. P., Beichman, C., Bryden, G., Downey, E. C., Durney, O., Esposito, S., Gaspar, A., Grenz, P., Haniff, C. A., Leisenring, J. M., Marion, L., McMahon, T. J., Millan-Gabet, R., Montoya, M., Morzinski, K. M., Pinna, E., Power, J., Puglisi, A., Roberge, A., Serabyn, E., Skemer, A. J., Stapelfeldt, K., Su, K. Y. L., Vaitheeswaran, V., and Wyatt, M. C., "The HOSTS Survey-Exozodiacal Dust Measurements for 30 Stars," The Astronomical Journal 155, 194 (May 2018). 
[35] Ertel, S., Defrère, D., Hinz, P., Mennesson, B., Kennedy, G. M., Danchi, W. C., Gelino, C., Hill, J. M., Hoffmann, W. F., Mazoyer, J., Rieke, G., Shannon, A., Stapelfeldt, K., Spalding, E., Stone, J. M., Vaz, A., Weinberger, A. J., Willems, P., Absil, O., Arbo, P., Bailey, V. P., Beichman, C., Bryden, G., Downey, E. C., Durney, O., Esposito, S., Gaspar, A., Grenz, P., Haniff, C. A., Leisenring, J. M., Marion, L., McMahon, T. J., Millan-Gabet, R., Montoya, M., Morzinski, K. M., Perera, S., Pinna, E., Pott, J.-U., Power, J., Puglisi, A., Roberge, A., Serabyn, E., Skemer, A. J., Su, K. Y. L., Vaitheeswaran, V., and Wyatt, M. C., "The HOSTS Survey for Exozodiacal Dust: Observational Results from the Complete Survey," The Astronomical Journal 159, 177 (Mar. 2020). Publisher: American Astronomical Society.

[36] Defrère, D., Hinz, P., Kennedy, G., Stone, J., Rigley, J., Ertel, S., Gaspar, A., Bailey, V., Hoffmann, W., Mennesson, B., Millan-Gabet, R., Danchi, W., Absil, O., Arbo, P., Beichman, C., Bonavita, M., Brusa, G., Bryden, G., Downey, E., Esposito, S., Grenz, P., Haniff, C., Hill, J., Leisenring, J., Males, J., McMahon, T., Montoya, M., Morzinski, K., Pinna, E., Puglisi, A., Rieke, G., Roberge, A., Rousseau, H., Serabyn, E., Spalding, E., Skemer, A., Stapelfeldt, K., Su, K., Vaz, A., Weinberger, A., and Wyatt, M., "The HOSTS Survey: Evidence for an Extended Dust Disk and Constraints on the Presence of Giant Planets in the Habitable Zone of beta Leo," Submitted to AJ. (2021, submitted to AJ).

[37] Mennesson, B. and the HabEx STDT team, "Habitable Exoplanet Observatory (HabEx)," https://www.jpl.nasa.gov/habex/ (2019).

[38] Roberge, A., Petersen, B., Fisher, D., and the LUVOIR STDT team, "LUVOIR Study," http://asd.gsfc.nasa.gov/luvoir/ (2019).

[39] Zhao, M., Monnier, J. D., Pedretti, E., Thureau, N., Mérand, A., Ten Brummelaar, T., McAlister, H., Ridgway, S. T., Turner, N., Sturmann, J., Sturmann, L., Goldfinger, P. J., and Farrington, C., "IMAGING AND MODELING RAPIDLY ROTATING STARS: alpha CEPHEI AND alpha OPHIUCHI," The Astrophysical Journal 701, 209-224 (Aug. 2009).

[40] Chelli, A., Duvert, G., Malbet, F., and Kern, P., "Phase closure nulling: Application to the spectroscopy of faint companions," Astronomy \& Astrophysics 498, 321-327 (Apr. 2009).

[41] Absil, O., Le Bouquin, J.-B., Lebreton, J., Augereau, J.-C., Benisty, M., Chauvin, G., Hanot, C., Mérand, A., and Montagnier, G., "Deep near-infrared interferometric search for low-mass companions around beta Pictoris," Astronomy and Astrophysics 520, L2 (Sept. 2010).

[42] Danchi, W. C., Rajagopal, J., Kuchner, M., Richardson, L. J., and Deming, D., "The Importance of Phase in Nulling Interferometry and a Three-Telescope Closure-Phase Nulling Interferometer Concept," The Astrophysical Journal 645, 1554-1559 (July 2006).

[43] Lachaume, R., "On marginally resolved objects in optical interferometry," Astronomy \& Astrophysics 400, 795-803 (Mar. 2003). Number: 2 Publisher: EDP Sciences.

[44] Monnier, J. D., Pedretti, E., Thureau, N., Berger, J.-P., Millan-Gabet, R., ten Brummelaar, T., McAlister, H., Sturmann, J., Sturmann, L., Muirhead, P., Tannirkulam, A., Webster, S., and Zhao, M., "Michigan Infrared Combiner (MIRC): commissioning results at the CHARA Array," in [Society of Photo-Optical Instrumentation Engineers (SPIE) Conference Series], Monnier, J. D., Schöller, M., and Danchi, W. C., eds., Society of Photo-Optical Instrumentation Engineers (SPIE) Conference Series 6268, 62681P (June 2006).

[45] Petrov, R. G., Malbet, F., Weigelt, G., Antonelli, P., Beckmann, U., Bresson, Y., Chelli, A., Dugué, M., Duvert, G., Gennari, S., Glück, L., Kern, P., Lagarde, S., Le Coarer, E., Lisi, F., Millour, F., Perraut, K., Puget, P., Rantakyrö, F., Robbe-Dubois, S., Roussel, A., Salinari, P., Tatulli, E., Zins, G., Accardo, M., Acke, B., Agabi, K., Altariba, E., Arezki, B., Aristidi, E., Baffa, C., Behrend, J., Blöcker, T., Bonhomme, S., Busoni, S., Cassaing, F., Clausse, J. M., Colin, J., Connot, C., Delboulbé, A., Domiciano de Souza, A., Driebe, T., Feautrier, P., Ferruzzi, D., Forveille, T., Fossat, E., Foy, R., Fraix-Burnet, D., Gallardo, A., Giani, E., Gil, C., Glentzlin, A., Heiden, M., Heininger, M., Hernandez Utrera, O., Hofmann, K. H., Kamm, D., Kiekebusch, M., Kraus, S., Le Contel, D., Le Contel, J. M., Lesourd, T., Lopez, B., Lopez, M., Magnard, Y., Marconi, A., Mars, G., Martinot-Lagarde, G., Mathias, P., Mège, P., Monin, J. L., Mouillet, D., Mourard, D., Nussbaum, E., Ohnaka, K., Pacheco, J., Perrier, C., Rabbia, Y., Rebattu, S., Reynaud, F., Richichi, A., Robini, A., Sacchettini, M., Schertl, D., Schöller, M., Solscheid, W., Spang, A., Stee, P., Stefanini, P., Tallon, M., Tallon-Bosc, I., Tasso, D., Testi, L., Vakili, F., von der Lühe, O., Valtier, J. C., 
Vannier, M., and Ventura, N., "AMBER, the near-infrared spectro-interferometric three-telescope VLTI instrument," $A \mathscr{E} A$ 464, 1-12 (Mar. 2007).

[46] Anugu, N., Le Bouquin, J.-B., Monnier, J. D., Kraus, S., Setterholm, B. R., Labdon, A., Davies, C. L., Lanthermann, C., Gardner, T., Ennis, J., Johnson, K. J. C., Ten Brummelaar, T., Schaefer, G., and Sturmann, J., "MIRC-X: A Highly Sensitive Six-telescope Interferometric Imager at the CHARA Array," AJ 160, 158 (Oct. 2020).

[47] Le Bouquin, J. B., Berger, J. P., Lazareff, B., Zins, G., Haguenauer, P., Jocou, L., Kern, P., Millan-Gabet, R., Traub, W., Absil, O., Augereau, J. C., Benisty, M., Blind, N., Bonfils, X., Bourget, P., Delboulbe, A., Feautrier, P., Germain, M., Gitton, P., Gillier, D., Kiekebusch, M., Kluska, J., Knudstrup, J., Labeye, P., Lizon, J. L., Monin, J. L., Magnard, Y., Malbet, F., Maurel, D., Ménard, F., Micallef, M., Michaud, L., Montagnier, G., Morel, S., Moulin, T., Perraut, K., Popovic, D., Rabou, P., Rochat, S., Rojas, C., Roussel, F., Roux, A., Stadler, E., Stefl, S., Tatulli, E., and Ventura, N., "PIONIER: a 4-telescope visitor instrument at VLTI," A\&3A 535, A67 (Nov. 2011).

[48] Kraus, S., "Star Formation and Fundamental Stellar Astrophysics with VLTI at 1 micrometer," in [The Very Large Telescope in 2030], 36 (July 2019).

[49] Szulágyi, J. and Ercolano, B., "Hydrogen Recombination Line Luminosities and Variability from Forming Planets," ApJ 902, 126 (Oct. 2020).

[50] Gravity Collaboration, Lacour, S., Nowak, M., Wang, J., Pfuhl, O., Eisenhauer, F., Abuter, R., Amorim, A., Anugu, N., Benisty, M., Berger, J. P., Beust, H., Blind, N., Bonnefoy, M., Bonnet, H., Bourget, P., Brandner, W., Buron, A., Collin, C., Charnay, B., Chapron, F., Clénet, Y., Coudé Du Foresto, V., de Zeeuw, P. T., Deen, C., Dembet, R., Dexter, J., Duvert, G., Eckart, A., Förster Schreiber, N. M., Fédou, P., Garcia, P., Garcia Lopez, R., Gao, F., Gendron, E., Genzel, R., Gillessen, S., Gordo, P., Greenbaum, A., Habibi, M., Haubois, X., Haußmann, F., Henning, T., Hippler, S., Horrobin, M., Hubert, Z., Jimenez Rosales, A., Jocou, L., Kendrew, S., Kervella, P., Kolb, J., Lagrange, A. M., Lapeyrère, V., Le Bouquin, J. B., Léna, P., Lippa, M., Lenzen, R., Maire, A. L., Mollière, P., Ott, T., Paumard, T., Perraut, K., Perrin, G., Pueyo, L., Rabien, S., Ramírez, A., Rau, C., Rodríguez-Coira, G., Rousset, G., Sanchez-Bermudez, J., Scheithauer, S., Schuhler, N., Straub, O., Straubmeier, C., Sturm, E., Tacconi, L. J., Vincent, F., van Dishoeck, E. F., von Fellenberg, S., Wank, I., Waisberg, I., Widmann, F., Wieprecht, E., Wiest, M., Wiezorrek, E., Woillez, J., Yazici, S., Ziegler, D., and Zins, G., "First direct detection of an exoplanet by optical interferometry. Astrometry and K-band spectroscopy of HR 8799 e," A\&A 623, L11 (Mar. 2019).

[51] Defrère, D., Absil, O., Berger, J.-P., Boulet, T., Danchi, W. C., Ertel, S., Gallenne, A., Hénault, F., Hinz, P., Huby, E., Ireland, M., Kraus, S., Labadie, L., Le Bouquin, J.-B., Martin, G., Matter, A., Mérand, A., Mennesson, B., Minardi, S., Monnier, J. D., Norris, B., de Xivry, G. O., Pedretti, E., Pott, J.-U., Reggiani, M., Serabyn, E., Surdej, J., Tristram, K. R. W., and Woillez, J., "The path towards high-contrast imaging with the VLTI: the Hi-5 project," Experimental Astronomy 46, 475-495 (Dec. 2018).

[52] Defrère, D., Ireland, M., Absil, O., Berger, J.-P., Danchi, W. C., Ertel, S., Gallenne, A., Hénault, F., Hinz, P., Huby, E., Kraus, S., Labadie, L., Le Bouquin, J.-B., Martin, G., Matter, A., Mennesson, B., Mérand, A., Minardi, S., Monnier, J. D., Norris, B., Orban de Xivry, G., Pedretti, E., Pott, J.-U., Reggiani, M., Serabyn, E., Surdej, J., Tristram, K. R. W., and Woillez, J., "Hi-5: a potential high-contrast thermal near-infrared imager for the VLTI," 0701, 107010U (July 2018).

[53] Fernandes, R. B., Mulders, G. D., Pascucci, I., Mordasini, C., and Emsenhuber, A., "Hints for a Turnover at the Snow Line in the Giant Planet Occurrence Rate," The Astrophysical Journal 874, 81 (Mar. 2019). arXiv: 1812.05569.

[54] de Kok, R. J., Birkby, J., Brogi, M., Schwarz, H., Albrecht, S., de Mooij, E. J. W., and Snellen, I. A. G., "Identifying new opportunities for exoplanet characterisation at high spectral resolution," Astronomy and Astrophysics 561, A150 (Jan. 2014).

[55] Kirchschlager, F., Ertel, S., Wolf, S., Matter, A., and Krivov, A. V., "First L band detection of hot exozodiacal dust with VLTI/MATISSE," Monthly Notices of the Royal Astronomical Society: Letters 499, L47-L52 (Nov. 2020). Publisher: Oxford Academic.

[56] Eisenhauer, F., "GRAVITY+: Towards faint science," in [The Very Large Telescope in 2030], 30 (July 2019). 
[57] Hinz, P. M., Defrère, D., Skemer, A., Bailey, V., Stone, J., Spalding, E., Vaz, A., Pinna, E., Puglisi, A., Esposito, S., Montoya, M., Downey, E., Leisenring, J., Durney, O., Hoffmann, W., Hill, J., Millan-Gabet, R., Mennesson, B., Danchi, W., Morzinski, K., Grenz, P., Skrutskie, M., and Ertel, S., "Overview of LBTI: a multipurpose facility for high spatial resolution observations," in [Optical and Infrared Interferometry and Imaging V], Malbet, F., Creech-Eakman, M. J., and Tuthill, P. G., eds., Society of Photo-Optical Instrumentation Engineers (SPIE) Conference Series 9907, 990704 (Aug. 2016).

[58] Ertel, S., Hinz, P. M., Vaz, A., Stone, J. M., Spalding, E. A., Wagner, K., Power, J., Maier, E. R., Montoya, O. M., West, G. S., Durney, O., Grenz, P., Leisenring, J., Defrère, D., Skemer, A. J., Mennesson, B., Kennedy, G., Hoffmann, W., Hill, J., Anugu, N., Pinna, E., Puglisi, A., Rossi, F., and Perera, S., "Overview and prospects of the LBTI beyond the completed HOSTS survey," SPIE, this series (2021, SPIE, this series).

[59] Stone, J. M., Skemer, A. J., Hinz, P. M., Bonavita, M., Kratter, K. M., Maire, A.-L., Defrere, D., Bailey, V. P., Spalding, E., Leisenring, J. M., Desidera, S., Bonnefoy, M., Biller, B., Woodward, C. E., Henning, T., Skrutskie, M. F., Eisner, J. A., Crepp, J. R., Patience, J., Weigelt, G., De Rosa, R. J., Schlieder, J., Brandner, W., Apai, D., Su, K., Ertel, S., Ward-Duong, K., Morzinski, K. M., Schertl, D., Hofmann, K.-H., Close, L. M., Brems, S. S., Fortney, J. J., Oza, A., Buenzli, E., and Bass, B., "The LEECH Exoplanet Imaging Survey: Limits on Planet Occurrence Rates under Conservative Assumptions," The Astronomical Journal 156, 286 (Dec. 2018).

[60] Hinz, P. M., Skemer, A., Stone, J., Montoya, O. M., and Durney, O., "Design of ALES: a broad wavelength integral field unit for LBTI/LMIRcam," in [Ground-based and Airborne Instrumentation for Astronomy VII], Evans, C. J., Simard, L., and Takami, H., eds., Society of Photo-Optical Instrumentation Engineers (SPIE) Conference Series 10702, 107023L (July 2018).

[61] Hoffmann, W. F., Hinz, P. M., Defrère, D., Leisenring, J. M., Skemer, A. J., Arbo, P. A., Montoya, M., and Mennesson, B., "Operation and performance of the mid-infrared camera, NOMIC, on the Large Binocular Telescope," in [Ground-based and Airborne Instrumentation for Astronomy V], Ramsay, S. K., McLean, I. S., and Takami, H., eds., Society of Photo-Optical Instrumentation Engineers (SPIE) Conference Series 9147, 91471 O (July 2014).

[62] Ertel, S., Defrère, D., Mennesson, B., Hinz, P., Willems, P., Perera, S., R., Z., the HOSTS science team, and the LBTI instrument team, "Final report on LBTI/HOSTS sensitivity study," https://nexsci.caltech.edu/missions/LBTI/ (2020).

[63] Cabrera, M. S., McMurtry, C. W., Dorn, M. L., Forrest, W. J., Pipher, J. L., and Lee, D., "Development of 13- $\mu \mathrm{m}$ cutoff HgCdTe detector arrays for astronomy," Journal of Astronomical Telescopes, Instruments, and Systems 5, 036005 (July 2019).

[64] Ertel, S., Kennedy, G. M., Defrère, D., Hinz, P., Shannon, A. B., Mennesson, B., Danchi, W. C., Gelino, C., Hill, J. M., Hoffman, W. F., Rieke, G., Spalding, E., Stone, J. M., Vaz, A., Weinberger, A. J., Willems, P., Absil, O., Arbo, P., Bailey, V. P., Beichman, C., Bryden, G., Downey, E. C., Durney, O., Esposito, S., Gaspar, A., Grenz, P., Haniff, C. A., Leisenring, J. M., Marion, L., McMahon, T. J., Millan-Gabet, R., Montoya, M., Morzinski, K. M., Pinna, E., Power, J., Puglisi, A., Roberge, A., Serabyn, E., Skemer, A. J., Stapelfeldt, K., Su, K. Y. L., Vaitheeswaran, V., and Wyatt, M. C., "The HOSTS survey for exo-zodiacal dust: preliminary results and future prospects," in [Space Telescopes and Instrumentation 2018: Optical, Infrared, and Millimeter Wave], Lystrup, M., MacEwen, H. A., Fazio, G. G., Batalha, N., Siegler, N., and Tong, E. C., eds., Society of Photo-Optical Instrumentation Engineers (SPIE) Conference Series 10698, 106981V (July 2018).

[65] Spalding, E., Hinz, P., Ertel, S., Maier, E., and Stone, J., "Towards controlled Fizeau observations with the Large Binocular Telescope," in [Optical and Infrared Interferometry and Imaging VI], Creech-Eakman, M. J., Tuthill, P. G., and Mérand, A., eds., Society of Photo-Optical Instrumentation Engineers (SPIE) Conference Series 10701, 107010J (July 2018).

[66] Spalding, E., Hinz, P., Morzinksi, K., Ertel, S., Grenz, P., Maier, E., Stone, J., and Vaz, A., "Status of commissioning stabilized infrared Fizeau interferometry with LBTI," in [Society of Photo-Optical Instrumentation Engineers (SPIE) Conference Series], Society of Photo-Optical Instrumentation Engineers (SPIE) Conference Series 11117, 111171S (Sept. 2019). 
[67] Defrère, D., Hinz, P., Downey, E., Ashby, D., Bailey, V., Brusa, G., Christou, J., Danchi, W. C., Grenz, P., Hill, J. M., Hoffmann, W. F., Leisenring, J., Lozi, J., McMahon, T., Mennesson, B., Millan-Gabet, R., Montoya, M., Powell, K., Skemer, A., Vaitheeswaran, V., Vaz, A., and Veillet, C., "Co-phasing the Large Binocular Telescope: status and performance of LBTI/PHASECam," in [Optical and Infrared Interferometry $I V]$, Rajagopal, J. K., Creech-Eakman, M. J., and Malbet, F., eds., Society of Photo-Optical Instrumentation Engineers (SPIE) Conference Series 9146, 914609 (July 2014).

[68] Pinna, E., Esposito, S., Hinz, P., Agapito, G., Bonaglia, M., Puglisi, A., Xompero, M., Riccardi, A., Briguglio, R., Arcidiacono, C., Carbonaro, L., Fini, L., Montoya, M., and Durney, O., "SOUL: the Single conjugated adaptive Optics Upgrade for LBT," in [Adaptive Optics Systems V], Marchetti, E., Close, L. M., and Véran, J.-P., eds., Society of Photo-Optical Instrumentation Engineers (SPIE) Conference Series 9909, 99093V (July 2016).

[69] Hinz, P., Esposito, S., Apai, D., Brusa, G., Close, L., Guyon, O., Hill, J., Males, J., Pinna, E., and Puglisi, A., "Toward visible wavelength coherent imaging with the LBT," in [Optical and Infrared Interferometry IV], Rajagopal, J. K., Creech-Eakman, M. J., and Malbet, F., eds., Society of Photo-Optical Instrumentation Engineers (SPIE) Conference Series 9146, 914605 (July 2014).

[70] Monnier, J. D., Kraus, S., Ireland, M. J., Baron, F., Bayo, A., Berger, J.-P., Creech-Eakman, M., Dong, R., Duchêne, G., Espaillat, C., Haniff, C., Hönig, S., Isella, A., Juhasz, A., Labadie, L., Lacour, S., Leifer, S., Merand, A., Michael, E., Minardi, S., Mordasini, C., Mozurkewich, D., Olofsson, J., Paladini, C., Petrov, R., Pott, J.-U., Ridgway, S., Rinehart, S., Stassun, K., Surdej, J., Brummelaar, T. t., Turner, N., Tuthill, P., Vahala, K., van Belle, G., Vasisht, G., Wishnow, E., Young, J., and Zhu, Z., "The planet formation imager," Experimental Astronomy 46, 517-529 (Dec. 2018).

[71] Rinehart, S. A., Savini, G., Holland, W., Absil, O., Defrère, D., Spencer, L., Leisawitz, D., Rizzo, M., Juanola-Paramon, R., and Mozurkewich, D., "The path to interferometry in space," in [Optical and Infrared Interferometry and Imaging V], 9907, 99070S, International Society for Optics and Photonics (Aug. 2016).

[72] Monnier, J., Aarnio, A., Absil, O., Anugu, N., Baines, E., Bayo, A., Berger, J.-P., Cleeves, L. I., Dale, D., Danchi, W., Wit, W. J. d., Defrère, D., Domagal-Goldman, S., Elvis, M., Froebrich, D., Gai, M., Gandhi, P., Garcia, P., Gardner, T., Gies, D., Gonzalez, J.-F., Gunter, B., Hoenig, S., Ireland, M., Jorgensen, A. M., Kishimoto, M., Klarmann, L., Kloppenborg, B., Kluska, J., Knight, J. S., Kral, Q., Kraus, S., Labadie, L., Lawson, P., Bouquin, J.-B. L., Leisawitz, D., Lightsey, E. G., Linz, H., Lipscy, S., MacGregor, M., Matsuo, H., Mennesson, B., Meyer, M., Michael, E. A., Millour, F., Mozurkewich, D., Norris, R., Ollivier, M., Packham, C., Petrov, R., Pueyo, L., Pope, B., Quanz, S., Ragland, S., Rau, G., Regaly, Z., Riva, A., Roettenbacher, R., Savini, G., Setterholm, B., Sewilo, M., Smith, M., Spencer, L., Brummelaar, T. t., Turner, N., Belle, G. v., Weigelt, G., and Wittkowski, M., "A Realistic Roadmap to Formation Flying Space Interferometry," Bulletin of the AAS 51 (Sept. 2019). Publisher: PubPub.

[73] Danchi, W. C., Barry, R. K., Lawson, P. R., Traub, W. A., and Unwin, S., "The Fourier-Kelvin Stellar Interferometer (FKSI): a review, progress report, and update," in [Optical and Infrared Interferometry], 7013, 70132Q, International Society for Optics and Photonics (July 2008).

[74] Danchi, W. C. and Barry, R. K., "The Fourier-Kelvin Stellar Interferometer (FKSI) - infrared detection and characterization of exozodiacal dust to super-earths: a progress report," in [Optical and Infrared Interferometry II], 7734, 77340M, International Society for Optics and Photonics (July 2010).

[75] Ollivier, M., Absil, O., Allard, F., Berger, J.-P., Bordé, P., Cassaing, F., Chazelas, B., Chelli, A., Chesneau, O., Coudé du Foresto, V., Defrère, D., Duchon, P., Gabor, P., Gay, J., Herwats, E., Jacquinod, S., Kern, P., Kervella, P., Le Duigou, J.-M., Léger, A., Lopez, B., Malbet, F., Mourard, D., Pelat, D., Perrin, G., Rabbia, Y., Rouan, D., Reiss, J.-M., Rousset, G., Selsis, F., Stee, P., and Surdej, J., "PEGASE, an infrared interferometer to study stellar environments and low mass companions around nearby stars," Experimental Astronomy 23, 403-434 (Mar. 2009).

[76] Lacour, S., Lapeyrère, V., Gauchet, L., Arroud, S., Gourgues, R., Martin, G., Heidmann, S., Haubois, X., and Perrin, G., "CubeSats as pathfinders for planetary detection: the FIRST-S satellite," in [Space Telescopes and Instrumentation 2014: Optical, Infrared, and Millimeter Wave], 9143, 91432N, International Society for Optics and Photonics (Aug. 2014).

[77] Hansen, J. T. and Ireland, M. J., "A Linear Formation Flying Astronomical Interferometer in Low Earth Orbit," arXiv:1912.02350 [astro-ph] (Apr. 2020). arXiv: 1912.02350. 
[78] Dandumont, C., Defrère, D., Kammerer, J., Absil, O., Quanz, S. P., and Loicq, J., "Exoplanet detection yield of a space-based Bracewell interferometer from small to medium satellites," Journal of Astronomical Telescopes, Instruments, and Systems 6, 035004 (Sept. 2020). Publisher: International Society for Optics and Photonics.

[79] Quanz, S. P., Meyer, M. R., Kenworthy, M., Kasper, M., Lenzen, R., Girard, J., Hinz, P., Geissler, K., Brander, W., Henning, T., and Wolf, S., "Direct detection of exoplanets and circumstellar disks using NaCo APP and NaCo PDI," (Oct. 2010).

[80] Quanz, S. P., Absil, O., Angerhausen, D., Benz, W., Bonfils, X., Berger, J.-P., Brogi, M., Cabrera, J., Danchi, W. C., Defrère, D., van Dishoeck, E., Ehrenreich, D., Ertel, S., Fortney, J., Gaudi, S., Girard, J., Glauser, A., Grenfell, J. L., Ireland, M., Janson, M., Kammerer, J., Kitzmann, D., Kraus, S., Krause, O., Labadie, L., Lacour, S., Lichtenberg, T., Line, M., Linz, H., Loicq, J., Mennesson, B., Meyer, M. R., Miguel, Y., Monnier, J., N’Diaye, M., Pallé, E., Queloz, D., Rauer, H., Ribas, I., Rugheimer, S., Selsis, F., Serabyn, G., Snellen, I., Sozzetti, A., Stapelfeldt, K. R., Triaud, A., Udry, S., and Wyatt, M., "Atmospheric characterization of terrestrial exoplanets in the mid-infrared: biosignatures, habitability \& diversity," arXiv:1908.01316 [astro-ph] Arxiv (Aug. 2019). arXiv: 1908.01316.

[81] Des Marais, D. J., Harwit, M. O., Jucks, K. W., Kasting, J. F., Lin, D. N., Lunine, J. I., Schneider, J., Seager, S., Traub, W. A., and Woolf, N. J., "Remote Sensing of Planetary Properties and Biosignatures on Extrasolar Terrestrial Planets," Astrobiology 2, 153-181 (June 2002). Publisher: Mary Ann Liebert, Inc., publishers.

[82] Léger, A., Defrère, D., Muñoz, A. G., Godolt, M., Grenfell, J., Rauer, H., and Tian, F., "Searching for Atmospheric Bioindicators in Planets around the Two Nearby Stars, Proxima Centauri and Epsilon Eridani-Test Cases for Retrieval of Atmospheric Gases with Infrared Spectroscopy," Astrobiology 19, 797810 (June 2019).

[83] Schwieterman, E. W., Kiang, N. Y., Parenteau, M. N., Harman, C. E., DasSarma, S., Fisher, T. M., Arney, G. N., Hartnett, H. E., Reinhard, C. T., Olson, S. L., Meadows, V. S., Cockell, C. S., Walker, S. I., Grenfell, J. L., Hegde, S., Rugheimer, S., Hu, R., and Lyons, T. W., "Exoplanet Biosignatures: A Review of Remotely Detectable Signs of Life," Astrobiology 18, 663-708 (May 2018). Publisher: Mary Ann Liebert, Inc., publishers.

[84] Line, M., Quanz, S. P., Schwieterman, E. W., Fortney, J. J., Stevenson, K. B., Greene, T., Zellem, R., Morley, C., Kataria, T., Tremblay, L., Mennesson, B., Iyer, A., Mawet, D., Iro, N., Kaltenegger, L., Defrere, D., Kite, E. S., Caldwell, D. A., Shkolnik, E., Dragomir, D., Ngo, H., Blecic, J., Lichtenberg, T., Angerhausen, D., Solmaz, A., Wolf, E. T., Monnier, J., Hicks, B., Kane, S. R., Danchi, W., Stassun, K., Valencia, D., and Staguhn, J., "The Importance of Thermal Emission Spectroscopy for Understanding Terrestrial Exoplanets," Vol. 51, Issue 3 (Astro2020 Science White Papers) 51 (May 2019). Publisher: PubPub.

[85] Lay, O. P., "Systematic errors in nulling interferometers," Applied Optics 43, 6100-6123 (Nov. 2004). Publisher: Optical Society of America.

[86] Lay, O. P., "Removing instability noise in nulling interferometers," in [Advances in Stellar Interferometry], 6268, 62681A, International Society for Optics and Photonics (June 2006).

[87] Martin, S., Booth, A., Liewer, K., Raouf, N., Loya, F., and Tang, H., "High performance testbed for fourbeam infrared interferometric nulling and exoplanet detection," Applied Optics 51, 3907-3921 (June 2012). Publisher: Optical Society of America.

[88] Defrère, D., Absil, O., and Beichman, C. A., "Interferometric Space Missions for Exoplanet Science: Legacy of Darwin/TPF," in [Handbook of Exoplanets], Deeg, H. J. and Belmonte, J. A., eds., 1229-1255, Springer International Publishing, Cham (2018).

[89] Defrère, D., Léger, A., Absil, O., Beichman, C., Biller, B., Danchi, W. C., Ergenzinger, K., Eiroa, C., Ertel, S., Fridlund, M., Muñoz, A. G., Gillon, M., Glasse, A., Godolt, M., Grenfell, J. L., Kraus, S., Labadie, L., Lacour, S., Liseau, R., Martin, G., Mennesson, B., Micela, G., Minardi, S., Quanz, S. P., Rauer, H., Rinehart, S., Santos, N. C., Selsis, F., Surdej, J., Tian, F., Villaver, E., Wheatley, P. J., and Wyatt, M., "Space-based infrared interferometry to study exoplanetary atmospheres," Experimental Astronomy 46, 543-560 (Dec. 2018). 
[90] Kopparapu, R. K., Hébrard, E., Belikov, R., Batalha, N. M., Mulders, G. D., Stark, C., Teal, D., DomagalGoldman, S., and Mandell, A., "Exoplanet Classification and Yield Estimates for Direct Imaging Missions," The Astrophysical Journal 856, 122 (Mar. 2018).

[91] Kammerer, J. and Quanz, S. P., "Simulating the exoplanet yield of a space-based mid-infrared interferometer based on Kepler statistics," Astronomy and Astrophysics 609, A4 (Jan. 2018). 